\title{
Fast Numerical Calculation of UHF Propagation over Two Dimensional Dielectric Irregular Terrain using an Integral Equation Method.
}

\author{
Eamonn M. Kenny and Peter J. Cullen† \\ The authors are with the Department of Electronic and Electrical Engineering, \\ Trinity College Dublin, Ireland. \\ $\dagger$ Author to whom all correspondence should be addressed. \\ e-mail: peter.cullen@tcd.ie
}

\begin{abstract}
In this paper we describe a new high speed method for the calculation of UHF propagation over irregular terrain which is considered to be a homogeneous dielectric. The method, given that it is rigorous and includes both forward and back scattering, is very fast. The antenna pattern, tilt etc. may be specified arbitrarily. As expected, results compare very well with measurements in the case when the terrain conductivity is high. Moreover, we explore the variation in the predicted fields with varying constitutive parameters.
\end{abstract}

\section{Introduction}

Whilst the treatment of terrain as perfectly electrically conducting, gives good results at UHF when compared to measurements [1], it is natural to consider the effect of finite conductivity. In this paper we build upon the recent development of fast integral equation techniques for electrically massive perfect electrical conductors by Moroney and Cullen [2] and similar work on large scatterers by Aberegg and Peterson [3], and James [10]. In particular we consider how the idea in [2] may be extended to the dielectric problem in two dimensions.

The fast electric field integral equation (EFIE) employed in [2] was developed by choosing a basis function based on the phase of the incident field which leads to a moment method with an impedance matrix of substantially reduced rank (compared to that obtainable using standard pulse basis functions). The required density of unknowns has been demonstrated to be as few as one every $50 \mathrm{~m}$ at a frequency of $1900 \mathrm{MHz}$, or roughly one unknown per 300 wavelengths.

In this paper our starting point is the full combined field integral equation CFIE [4]. The combination of the basis functions with the CFIE is not a trivial task, and will be discussed later in depth. The numerical formulation of the high speed CFIE will be thoroughly described. When calculating elements of the kernel (moment) matrix there are two main types of approximation to consider. The forward scattering integrals are approximated by quadrature rules while the back scattering integrals are approximated by asymptotic expansions, due to the fact that the former terms results in slowly varying functions while the latter results in a quickly varying function which cancels almost everywhere. Also, derivatives must be approximated by backward differencing so that the method remains implicit.
For the purpose of verification it is important to consider three cases, namely: good dielectrics, lossy dielectrics and good conductors. The different cases are defined using a well known dimensionless quantity defined as the electric loss tangent (see Balanis [5]). The case of good conductors is easily verified since this is directly comparable with [2]. In the other cases the results are not easily verified on massive problems since it appears that no other practical solution technique exists. For the other cases we can verify our method using a known solution to the scattering from canonical problems such as a flat plate.

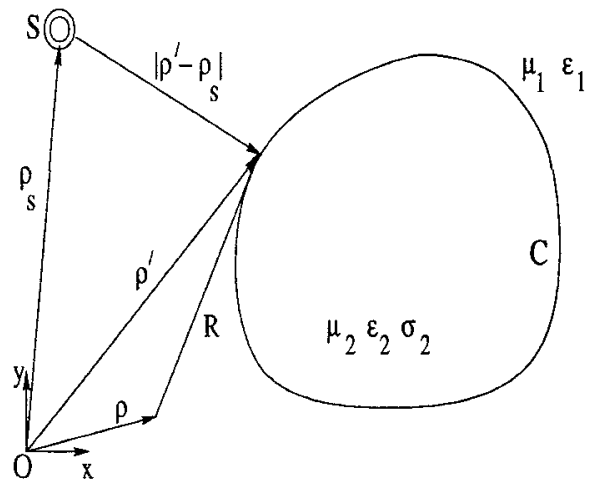

Fig. 1. The geometry of the problem, the source $\mathrm{S}$, contour $\mathrm{C}$ and origin O.

\section{Formulation of method}

The formulation which follows is outlined for a twodimensional scattering medium which is lossy, linear, homogeneous, non-dispersive and isotropic embedded in free space with fields oriented as transverse magnetic in $z$.

Starting with a well known CFIE formulation [4], the objective is to adapt this method to find a numerically efficient solution for irregular terrain by introducing a complex basis function $[2,3]$ and solving the problem by use of the point collocation method [2].

It will suffice to begin with the CFIE equations of [4] namely (1) and (2), as a way to develop the fast CFIE. The equations are defined as follows:

$$
\begin{aligned}
& E_{z}^{i}(\rho)=\int_{C}\left[J_{z}\left(\rho^{\prime}\right) G_{1}\left(\rho, \rho^{\prime}\right)+M_{s}\left(\rho^{\prime}\right) G_{2}\left(\rho, \rho^{\prime}\right)\right] d \rho^{\prime}(1) \\
& H_{s}^{i}(\rho)=\int_{C}\left[J_{z}\left(\rho^{\prime}\right) G_{3}\left(\rho, \rho^{\prime}\right)+M_{s}\left(\rho^{\prime}\right) G_{4}\left(\rho, \rho^{\prime}\right)\right] d \rho^{\prime}
\end{aligned}
$$




$$
+\frac{\partial}{\partial s} \int_{C}\left[\frac{\partial}{\partial s^{\prime}} M_{s}\left(\rho^{\prime}\right)\right] G_{4}^{\prime}\left(\rho, \rho^{\prime}\right) d \rho^{\prime}
$$

where $E_{z}^{i}$ is the incident electric field, $H_{s}^{i}$ is the incident magnetic field, $J_{z}$ is the electric current, $M_{s}$ is the magnetic current, $\rho$ is an observation point on the contour $C, \rho^{\prime}$ is an integration point, $s$ is the arc length along the contour $C$ and the components are defined as:

$$
\begin{aligned}
& G_{1}\left(\rho, \rho^{\prime}\right)=\frac{\omega \mu_{1}}{4} H_{1}+\frac{\omega \mu_{2}}{4} H_{2} \\
& G_{2}\left(\rho, \rho^{\prime}\right)=\frac{\Phi^{\prime}(x, y)}{4 j R}\left[k_{1} H_{1}^{\prime}+k_{2} H_{2}^{\prime}\right] \\
& G_{3}\left(\rho, \rho^{\prime}\right)=\frac{\Phi(x, y)}{4 j R}\left[k_{1} H_{1}^{\prime}+k_{2} H_{2}^{\prime}\right] \\
& G_{4}\left(\rho, \rho^{\prime}\right)=\cos \left(\Omega-\Omega^{\prime}\right)\left[\frac{\omega \epsilon_{1}}{4} H_{1}+\frac{\omega \epsilon_{2}^{\prime}}{4} H_{2}\right] \\
& G_{4}^{\prime}\left(\rho, \rho^{\prime}\right)=\frac{1}{4 \omega \mu_{1}} H_{1}+\frac{1}{4 \omega \mu_{2}} H_{2}
\end{aligned}
$$

where

$$
\begin{aligned}
R & =\left|\rho-\rho^{\prime}\right| \\
\epsilon_{2}^{\prime} & =\epsilon_{2}\left(1-j \frac{\sigma}{\omega \epsilon_{2}}\right), \quad k_{2}=\sqrt{\omega^{2} \mu_{2} \epsilon_{2}^{\prime}} \\
H_{1} & =H_{0}^{(2)}\left(k_{1} R\right), \quad H_{2}=H_{0}^{(2)}\left(k_{2} R\right) \\
H_{1}^{\prime} & =H_{0}^{(2)^{\prime}}\left(k_{1} R\right), \quad H_{2}^{\prime}=H_{0}^{(2)^{\prime}}\left(k_{2} R\right) \\
\Phi(x, y) & =\left[\left(x-x^{\prime}\right) \cos \Omega+\left(y-y^{\prime}\right) \sin \Omega\right] \\
\Phi^{\prime}(x, y) & =\left[\left(x-x^{\prime}\right) \cos \Omega^{\prime}+\left(y-y^{\prime}\right) \sin \Omega^{\prime}\right]
\end{aligned}
$$

where $H_{0}^{(2)}$ is the hankel function of the second kind, $H_{0}^{(2)^{\prime}}$ is the derivative of hankel function of the second kind, $\epsilon_{1}, \epsilon_{2}$ are the permittivities on region 1 and $2, \mu_{1}, \mu_{2}$ are the permeabilities, $\epsilon_{2}^{\prime}$ is the effective permittivity, $k_{1}$ is the real phase constant of region $1, k_{2}$ is the complex phase constant of region $2, \omega$ is the angular frequency, $\Omega$ is the angle that a point $\rho$ not necessarily on $C$ makes with the positive $\mathrm{x}$-axis, and $\sigma_{2}$ is the conductivity of region 2 (See Fig. 1).

A point collocation method describes a discrete set of equations which are obtained by expressing the current $J_{z}$ and $M_{s}$ as piecewise polynomials each with $N$ unknowns. So, the currents $J_{z}$ and $M_{s}$ are expressed as independent basis sets, as follows:

$$
\begin{array}{r}
J_{z}\left(\rho^{\prime}\right)=\sum_{n=1}^{N} I_{n} g_{n}\left(\rho^{\prime}\right) \\
M_{s}\left(\rho^{\prime}\right)=\sum_{n=N+1}^{2 N} I_{n} g_{n}\left(\rho^{\prime}\right)
\end{array}
$$

where $I_{n}$ is the unknown currents and $g_{n}\left(\rho^{\prime}\right)$ is a chosen basis function. As in the case of [2], the problem can then be solved numerically, once a suitable choice of basis function is assumed. Substituting (8) into (1) and (2) results in a set of equations defined as the point-matching (collocation) method.

The problem arising from all conventional collocation methods, is that the discretization interval for a satisfactory solution must be at: least one quarter of the wavelength $(\lambda / 4)$ shown in [6]. This sampling rate is infeasible for most realistic propagation problems and hence the use of a fast CFIE is necessary for any reasonably sized problem. The approach therefore is to remove the quickly varying phase of the problem by the use of a suitable basis $g_{n}$. In [2] a method is described whereby the phase of the incident field can be used as a basis. This method assumes that the phase of the incident field approximates the phase of the current $J_{z}$ for the EFIE. Assuming this result is true for the CFIE, the suitable basis function is defined by:

$$
g_{n}\left(\rho^{\prime}\right)=p_{n} e^{-j k_{1}\left|\rho_{n}^{\prime}-\rho_{s}\right|}
$$

where $p_{n}$ is the pulse basis function defined on the interval $\mathcal{I}_{n}=\left[\rho_{n}^{\prime}-\Delta \rho_{n}^{\prime}, \rho_{n}^{\prime}+\Delta \rho_{n}^{\prime}\right]$, and $\rho_{s}$ is the position of the antenna relative to the origin. These points are positioned in the center of each piecewise line segment to preserve the consistency of the problem. It is unwise to position a match point at a turning point, since this will affect the stability of the results obtained from such a method, since there will occur a severe change in slope within the region of integration. If points are positioned at turning points, then $p_{n}$ must be a quadratic basis function.

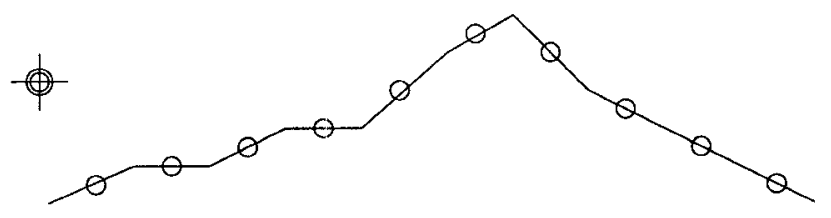

Fig. 2. Match point locations.

For a collocation method with $N$ match-points $(2 N$ simultaneous equations) the pulse basis functions are defined as:

$$
p_{n}(\rho)= \begin{cases}1 & \text { if } \rho \in \mathcal{I}_{n} \\ 0 & \text { otherwise }\end{cases}
$$

Note also that $p_{n}=p_{n+N}$ for $n=1,2, \cdots, N$, assuming that the magnetic current integration points are located at the same points as the electric current integration points. This is a different orientation to that of [4], and was found to have no ill effects. In fact, it is necessary for the matchpoints to be chosen in this manner, if the fast CFIE is to be applied including the pulse basis function $p_{n}$ for the reasons stated earlier. Fig. 2 shows the required positioning of these points for a sample terrain profile. 


\section{Numerical algorithm}

The resulting system obtained by the substitution of (9) into (1) to (2) is defined as follows:

$$
\left[\begin{array}{ll}
A & B \\
C & D
\end{array}\right]_{2 N \times 2 N}\left[\begin{array}{c}
I_{1} \\
I_{2}
\end{array}\right]=\left[\begin{array}{c}
E_{z}^{i} \\
M_{s}^{i}
\end{array}\right]
$$

where

$$
\begin{aligned}
A_{m n} & =\sum_{i=1}^{N_{n}} h_{n}\left[\frac{\omega \mu_{1}}{4} H_{1}+\frac{\omega \mu_{2}}{4} H_{2}\right] g_{n}\left(\rho_{n, i}^{\prime}\right) \\
B_{m n} & =\sum_{i=1}^{N_{n}} h_{n} \frac{\Phi_{n, i}^{\prime}}{4 j R}\left[k_{1} H_{1}^{\prime}+k_{2} H_{2}^{\prime}\right] g_{n}\left(\rho_{n, i}^{\prime}\right) \\
C_{m n} & =\sum_{i=1}^{N_{n}} h_{n} \frac{\Phi_{n, i}}{4 j R}\left[k_{1} H_{1}^{\prime}+k_{2} H_{2}^{\prime}\right] g_{n}\left(\rho_{n, i}^{\prime}\right) \\
D_{m n} & =\sum_{i=1}^{N_{n}} \frac{h_{n} g_{n}\left(\rho_{n, i}^{\prime}\right)}{4}\left\{\left[\frac{H_{3}}{\omega \mu_{1}}+\frac{H_{4}}{\omega \mu_{2}}\right] \frac{1}{4 h_{m} h_{n}}\right. \\
& \left.+\left[\omega \epsilon_{1} H_{1}+\omega \epsilon_{2}^{\prime} H_{2}\right] \cos \left(\Omega_{m}-\Omega_{n, i}^{\prime}\right)\right\}
\end{aligned}
$$

where

$$
\begin{aligned}
\rho_{m} & =\left(x_{m}, y_{m}\right)=m^{t h} \text { match-point centered in } \mathcal{I}_{m} \\
\rho_{n, i} & =\left(x_{n, i}, y_{n, i}\right)=i^{t h} \text { integration point of } \mathcal{I}_{n} \\
R & =\left|\rho_{m}-\rho_{n, i}^{\prime}\right| \\
R_{1} & =\left|\left(\rho_{m}+h_{m}\right)-\rho_{n, i-1}^{\prime}\right| \\
R_{2} & =\left|\left(\rho_{m}-h_{m}\right)-\rho_{n, i-1}^{\prime}\right| \\
R_{3} & =\left|\left(\rho_{m}+h_{m}\right)-\rho_{n, i+1}^{\prime}\right| \\
R_{4} & =\left|\left(\rho_{m}-h_{m}\right)-\rho_{n, i+1}^{\prime}\right| \\
h_{m} & =\text { The integration step within } \mathcal{I}_{m} \\
H_{3} & =H_{0}^{(2)}\left(k_{1} R_{1}\right)-H_{0}^{(2)}\left(k_{1} R_{2}\right) \\
& -H_{0}^{(2)}\left(k_{1} R_{3}\right)+H_{0}^{(2)}\left(k_{1} R_{4}\right) \\
H_{4} & =H_{0}^{(2)}\left(k_{2} R_{1}\right)-H_{0}^{(2)}\left(k_{2} R_{2}\right) \\
& -H_{0}^{(2)}\left(k_{2} R_{3}\right)+H_{0}^{(2)}\left(k_{2} R_{4}\right) \\
\Phi_{n, i}^{\prime} & =\left[\left(x_{m}-x_{n}^{\prime}\right) \cos \Omega_{n, i}^{\prime}+\left(y_{m}-y_{n, i}^{\prime}\right) \sin \Omega_{n, i}^{\prime}\right] \\
\Phi_{n, i} & =\left[\left(x_{m}-x_{n}^{\prime}\right) \cos \Omega_{n, i}+\left(y_{m}-y_{n, i}^{\prime}\right) \sin \Omega_{n, i}\right]
\end{aligned}
$$

The above integrals can be approximated by an Euler rule. The partial derivatives of the last term $D_{m n}$ can be approximated either numerically or analytically. In this case the analytical derivatives would result in a $H_{1}^{(2)}$ term which is singular divided by the distance $R$ which results in a function which is complicated to integrate. For this reason the derivatives are calculated numerically by central difference formulates. No instability will occur since central difference formulae are implicit methods and hence stable (see Lambert [7]).
The off-diagonal terms are calculated assuming hankel function approximations as in the following case:

$$
H_{0}^{(2)}\left(k_{1} R\right)=\sqrt{\frac{2}{\pi k_{1} R}} e^{-j\left(k_{1} R-\frac{\pi}{4}\right)}
$$

where it is assumed that the far field approximation of the hankel function $H_{0}^{(2)}$ is valid at any point where $m \neq n$. In the case of the self-terms it is necessary to avoid the singularity $\left(Y_{0}^{(2)}(0)=\infty\right)$. This is achieved by use of a well known log expansion [5].

$$
H_{0}^{(2)}(k \rho) \stackrel{k \rho \rightarrow 0}{\approx} 1-j \frac{2}{\pi} \ln \left(\frac{1.781 k \rho}{2}\right)
$$

which when numerically integrated gives

$$
\int_{-\frac{h_{n}}{2}}^{\frac{h_{n}}{2}} H_{0}^{(2)}(k \rho) d \rho=h_{n}\left[1-j \frac{2}{\pi} \ln \left(\frac{1.781 k h_{n}}{4 e}\right)\right]
$$

When considering the back-scattering contribution to the terms of the matrix, it is not necessary to calculate the integrals by an euler method. An asymptotic expansion with the first two leading terms will suffice. This will speed up the computation of the matrix filling considerably.

\section{Numerical results}

Choosing a pulse basis function $g_{n}=p_{n}$, it can be shown as in [6] that a moment method requires a point-match every quarter of a wavelength $(\lambda / 4)$ for satisfactory numerical convergence. Choosing the phase of the incident field as a basis function as in (9), it can be shown that the terms of the matrix kernel as defined by (12)-(15) are slowly varying in amplitude. This is very important, since the integrals can then be approximated by something as simple as an trapezoidal rule. Since the integrals are slowly varying, a discretization rate as high as $50 \lambda$ or $80 \lambda$ is possible. This means that, exact numerical computations can be used to obtain electric current, and scattered and total fields. Previously the size of the matrices involved, made it impossible to calculate UHF solutions over terrain of a few kilometres in length. Until recently only forward propagation solutions were possible for exact result calculations and required at least a day of computational time. With this CFIE method it is now possible to calculate the path-loss for undulating terrain in less than a few minutes.

When choosing examples, it is imperative that a wide variety of results be presented which will confirm convergence and stability of the outlined method. Three cases worth consisting spring to mind: good dielectrics, lossy dielectrics and good conductors. The dimensionless quantity $\tau$ namely the electric loss tangent describes these 3 classes of problems. We define $\tau$ as follows:

$$
\tau=\left(\frac{\sigma}{\omega \epsilon_{2}}\right)^{2}
$$


Upon definition of $\tau$, the problem can be broken into the three categories:

$$
\begin{array}{cc}
\tau \ll 1 & \text { Good dielectrics } \\
\tau=1 & \text { Lossy dielectrics } \\
\tau \gg 1 & \text { Good conductors }
\end{array}
$$

Results are now presented for the flat plate problem and for various slowly undulating danish terrain profiles.

\section{A. The Flat plate}

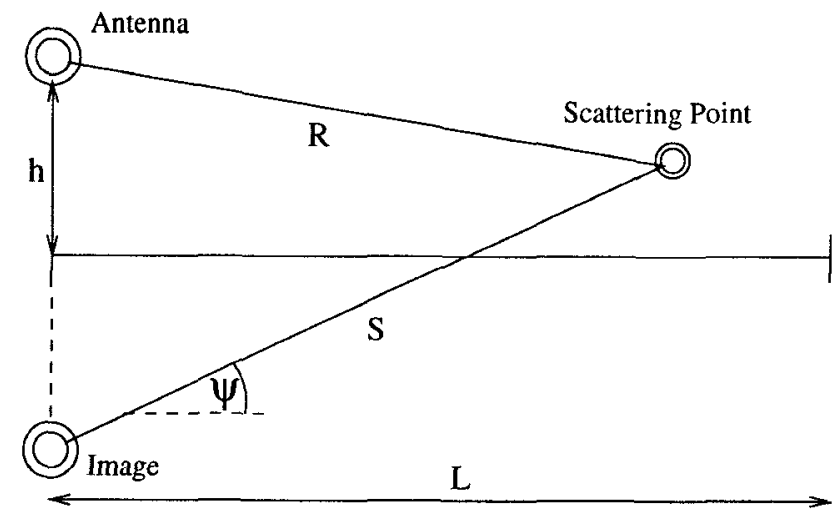

Fig. 3. The flat plate configuration

The results calculated over a flat plate are compared with the approximate solution defined by Clemmow [8] (See Fig. 3 for a diagramatic representation of the region). The scattered field formula is defined by:

$$
E_{s}=\left[-1+\frac{2 \sin \psi}{\sin \psi+\sqrt{\frac{\epsilon_{2}^{\prime}}{\epsilon_{1}}-\cos ^{2} \psi}}\right] H_{0}^{2}\left(k_{1} S\right)
$$

Fig. 4 shows the results obtained for a typical dry soil problem. The chosen constitutive parameters are $\sigma=$ $9.8 \times 10^{-3}, \epsilon_{2}^{R}=2.8, \tau=7 \times 10^{-2}, f=900 M H z, h=10.4$ and $L=5000$. It can be seen that there is a slight discrepancy between the solutions, in the region were they fall off quickly. This can be corrected by adaptive meshing about this region. The number of point-matches required by a conventional CFIE method with a point-match every one quarter wavelength would be 60,000 points. In this case we assume a point match every 50 metres (100 points). This result was calculated in 13.2 minutes on a $125 \mathrm{MHz}$ Power PC. Note that the results are identical to that of the PEC case.

To obtain results which deviate from that of the PEC solution, the constitutive parameters are chosen to be $\sigma=$ $9.8 \times 10^{-6}, \epsilon_{2}^{R}=1.001, \tau=1.92 \times 10^{-4}, f=900 \mathrm{MHz}$, $h=10.4$ and $L=5000$. The result in Fig. 5 deviated by about 4 decibels from the PEC result.

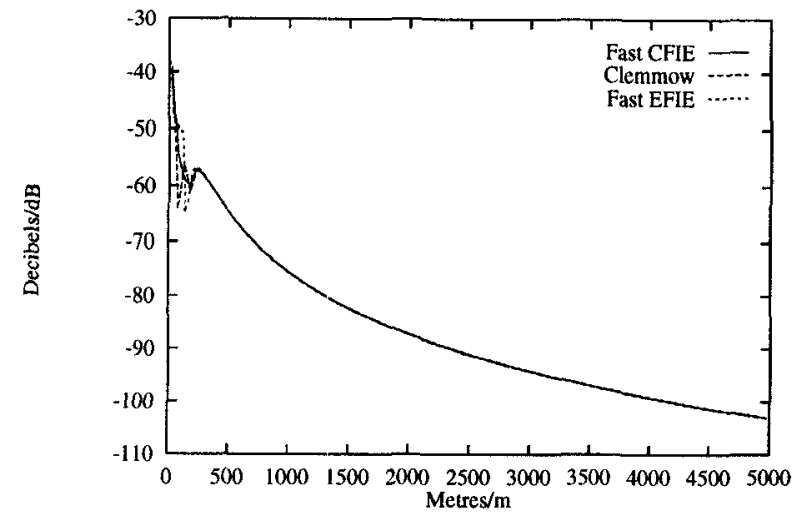

Fig. 4. Flat plate solution with $f=900 M H z, \sigma=9.8 \times 10^{-3}, \epsilon_{2}^{R}=2.8$.

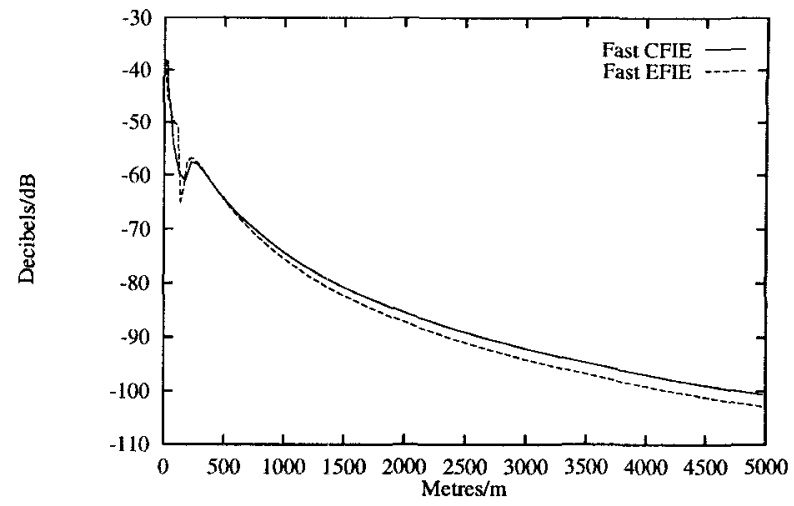

Fig. 5. Flat plate solution with $f=900 M H z, \sigma=9.8 \times 10^{-6}, \epsilon_{2}^{R}=1.001$

\section{B. Terrain Results}

We consider slowly undulating terrain profiles, obtained from [1]. The chosen constitutive parameters are $\sigma=$ $9.8 \times 10^{-3}, \epsilon_{2}^{R}=2.8, f=900 M H z$ and $h=10.4$. The point-matching rate was chosen to be every 50 metres. Fig. 6 shows the results obtained. It can be seen that the results are almost identical to that of the PEC case. The calculation required 15.7 minutes of computation on a $125 \mathrm{MHz}$ Power PC. Results are also presented for the case were the solution deviates from the PEC result (See Fig. 7). The method has been concluded to be convergent based on the assumption that continually halving the point-matching and integration discretization leads to a solution which is both numerically stable and consistent.

For the Jerslev terrain profile in Fig. 6, the time for computation of the result is less than 10 seconds using the method of [2]. Fig. 8 shows that the EFIE is quite sufficient for obtaining results in agreement with the measurement data. The EFIE result at $970 \mathrm{MHz}$ was calculated in 6 seconds. All results contain a $\frac{1}{\sqrt{R}}$ correction allowing for conversion from the $2 \mathrm{D}$ problem to the $3 \mathrm{D}$ problem. 


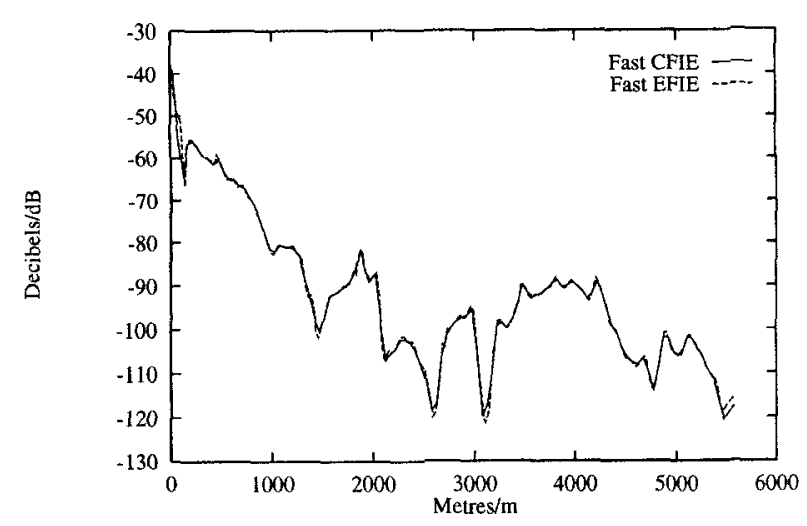

Fig. 6. Jerslev profile with $f=900 M H z, \sigma=9.8 \times 10^{-3}, \epsilon_{2}^{R}=2.8$.

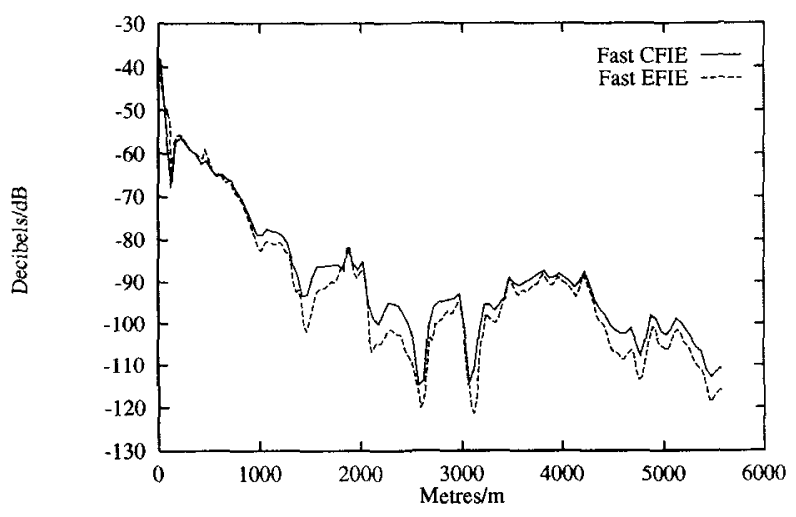

Fig. 7. Jerslev profile with $f=900 M H z, \sigma=9.8 \times 10^{-6}, \epsilon_{2}^{R}=1.001$.

\section{Conclusions}

Clearly, for realistic values of $\epsilon_{2}^{R}$ and $\sigma$ at UHF the CFIE agrees (almost perfectly) with the PEC method and the latter should be used since the speed of computation is far superior.

At lower frequencies this may not be the case. Only by using unrealistic values of $\epsilon_{2}^{R}$ can we obtain a significant difference in path-loss. This shows that deviations between measurements and predictions for terrain are due mainly to topographical factors such as the neglect of surface roughness and buildings and vegetation. In cases where the terrain is very irregular lateral variation in terrain height and scattering are also important.

There is really no point in using the complex permittivity in the CFIE as our empirical fit parameters, if this dubious procedure is to be applied. It makes more sense to use the impedance integral equation (Jones [11]) where the natural basis method of [2] can also be used.

\section{Acknowledgements}

The authors wish to express their gratitude to Prof. J. Bach Andersen for permission to use his measurements [1].

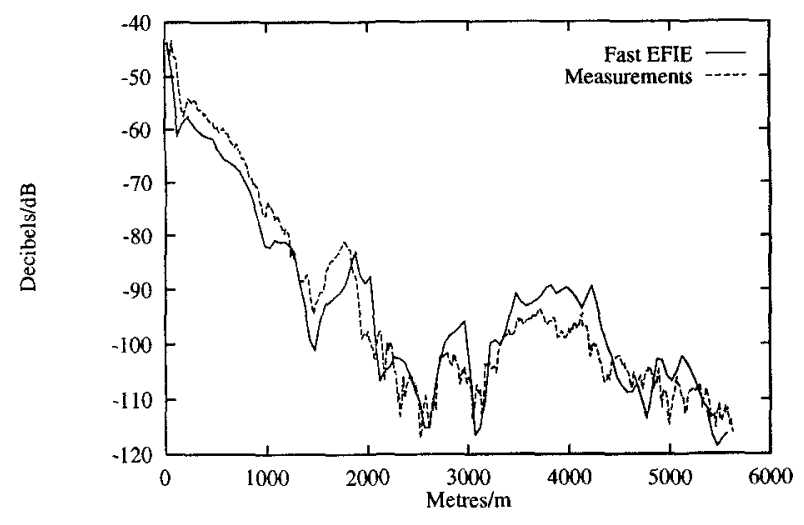

Fig. 8. Jerslev terrain profile with $f=970 M H z$

\section{References}

[1] J. B. Andersen, University of Aalborg: Personal Communication, Published IEEE AP, Jan. 1995, (1993).

[2] D. T. Moroney and P. J. Cullen, A Fast Integral Equation Approach to UHF Coverage Estimation, In Mobile and Personal Communications, Ed. E. Del Re, Elsevier, 1995.

[3] K. R. ABerega and A. F. Peterson, Integral Equation Asymptotic Phase Method to TwoDimensional Scattering, IEEE Trans. Antennas and Prop., Vol.43, No.5, 1995.

[4] K. Umashankar and A. Taflove, Computational Electromagnetics. Artech House, 1993.

[5] C. A. Balanis, Advanced Engineering Electromagnetics, Wiley, 1989.

[6] R. C. Hansen, ed., Moment Methods in Antennas and Scattering. Artech, Boston London, 1990.

[7] J. D. LAMBERT, Numerical Methods for Ordinary Differential Systems - The initial value problem. Wiley, 1991.

[8] P. C. Clemmow, The Plane Wave Spectrum Representation of Electromagnetics Fields, pp. 98-104, 1966.

[9] D. T. Moroney, Computational Methods for the Calculation of Electromagnetic Scattering from Large-Scale Perfect Electrical Conductors. PhD Thesis, University of Dublin, Trinity College, 1995.

[10] R. M. JAMES," A contribution to scattering calculation for small wave lengths-the high frequency panel method", IEEE. Trans. Antennas Propagation., vol 38, pp. 1625-1630, Oct 1990.

[11] D. S. JoNES, Methods in Electromagnetic Wave Propagation, Clarendon Press, Oxford, 1979. 